\title{
DYNAMICAL INSTABILITY OF LINEAR GANONICAL SYSTEMS
}

\author{
W. A. COPPEL and A. HOWE
}

(Received 21 March 1966)

In the present paper we obtain first approximation formulae for the regions of dynamical instability of linear canonical systems. These formulae are analogous to the formulae for Hamiltonian systems stated by Kreln and Jakubovic [5] and proved by Pittel' and Juzefovič [8]. Special cases were considered by Malkin [7] and Jakubovič [3]. Related papers are Hale [2] and Jakubovič [4]. However, our method differs from the methods used by these authors and seems to us to be both simpler and more general.

We consider the linear canonical system

$$
J d x / d t=\left[H_{0}+\varepsilon H(\Omega t, \varepsilon)\right] x,
$$

where $H_{0}$ is a constant Hermitian matrix, $H(\tau, \varepsilon)$ is a continuous Hermitian matrix function with period $2 \pi$ in $\tau$, and $J$ is an invertible skew-Hermitian matrix. Without loss of generality we can take

$$
J=i\left(\begin{array}{lr}
I_{p} & 0 \\
0 & -I_{q}
\end{array}\right)
$$

where $p$ and $q$ are positive integers such that $p+q=n$.

The unperturbed system

$$
J d x / d t=H_{0} x
$$

is assumed to be stable. Consequently, as shown in [1], the matrix $J^{-1} H_{0}$ has pure imaginary eigenvalues $i \omega_{1}, \cdots, i \omega_{n}$ and corresponding eigenvectors $f_{1}, \cdots, f_{n}$ such that

$$
i^{-1}\left(J f_{j}, f_{k}\right)=\left\{\begin{aligned}
0 & \text { if } j \neq k, \\
1 & \text { if } j=k \leqq p, \\
-1 & \text { if } j=k>p .1
\end{aligned}\right.
$$

The autonomous system (2) is strongly stable with respect to perturbations of period $2 \pi / \Omega_{0}$ if and only if

$$
\omega_{j} \not \equiv \omega_{k}\left(\bmod \Omega_{0}\right) \text { for } 1 \leqq j \leqq p<k \leqq n .
$$

1 Here $(x, y)$ denotes the usual scalar product $y^{*} x$. 
If this condition is not satisfied the question arises, for what values of $(\Omega, \varepsilon)$ near $\left(\Omega_{0}, 0\right)$ is the system (1) unstable?

The simplest case is where

$$
\omega_{j} \equiv \omega_{k}\left(\bmod \Omega_{0}\right) \quad(j \leqq p<k)
$$

holds for $j=j_{0}, k=k_{0}$ but for no other values of $j, k$. We shall show that in this case, if $\Omega=\Omega_{0}+\chi \varepsilon$, then the system (1) is unstable or stable for all small $\varepsilon \neq 0$ according as $\chi$ lies inside or outside a certain interval $\left(\chi_{1}, \chi_{2}\right)$. Thus the region in the $(\Omega, \varepsilon)$-plane for which $(1)$ is unstable is given to a first approximation near the point $\left(\Omega_{0}, 0\right)$ by the region between the lines with slopes $\chi_{1}, \chi_{2}$ through this point.

Our starting-point is the following lemma, which was suggested by a paper of Levinson [6].

Lemma. Suppose the matrix function $A(\varepsilon)$ is differentiable at $\varepsilon=0$. Let $A(0)$ have an $r$-fold eigenvalue $\lambda$ and let there exist $r$ linearly independent corresponding eigenvectors. Then for all small $\varepsilon \neq 0 A(\varepsilon)$ has $r$ eigenvalues of the form $\lambda+\varepsilon \mu_{j}+o(\varepsilon)(j=1, \cdots, r)$.

Proof. We can find an invertible matrix $C$ such that

$$
C^{-1} A(0) C=\left(\begin{array}{ll}
\lambda I_{r} & 0 \\
0 & Q
\end{array}\right)
$$

where $Q-\lambda I_{n-r}$ is non-singular. Hence

$$
C^{-1} A(\varepsilon) C=\left(\begin{array}{ll}
\lambda I_{r} & 0 \\
0 & Q
\end{array}\right)+\varepsilon B+o(\varepsilon),
$$

where $B=C^{-1} A^{\prime}(0) C$. Thus $w=\lambda+\varepsilon \mu$ is an eigenvalue of $A(\varepsilon)$ if and only if the determinant

$$
\left|\begin{array}{ll}
B_{11}-\mu I_{r}+o(1) & B_{12}+o(1) \\
\varepsilon B_{21}+o(\varepsilon) & Q-(\lambda+\varepsilon \mu) I_{n \rightarrow+}+\varepsilon B_{22}+o(\varepsilon)
\end{array}\right|
$$

vanishes, i.e. if and only if

$$
\operatorname{det}\left(B_{11}-\mu I_{r}\right) \operatorname{det}\left(Q-\lambda I_{n-r}\right)+f(\varepsilon, \mu)=0 \text {, }
$$

where $f(\varepsilon, \mu)$ is a polynomial of degree $n$ in $\mu$, the coefficients of which are functions of $\varepsilon$ tending to zero as $\varepsilon \rightarrow 0$. It follows at once from Rouchés theorem that if $B_{11}$ has eigenvalues $\mu_{1}, \cdots, \mu_{r}$ then $A(\varepsilon)$ has eigenvalues $\lambda+\varepsilon \mu_{j}+o(\varepsilon)(j=1, \cdots, r)$.

Putting $\tau=\Omega t$ and $\Omega=\Omega_{0}+\chi \varepsilon$, the system (1) takes the form

$$
x^{\prime}=\left(\Omega_{0}+\chi \varepsilon\right)^{-1} J^{-1}\left[H_{0}+\varepsilon H(\tau, \varepsilon)\right] x,
$$


where the prime denotes differentiation with respect to $\tau$. Let $X(\tau, \varepsilon)$ be the fundamental matrix for (3) such that $X(0, \varepsilon)=I$. In particular,

$$
X(\tau, 0)=e^{\tau \Omega_{0}^{-1} J^{-1} H_{0}} .
$$

By standard theorems on the differentiability of solutions with respect to a parameter, $X(\tau, \varepsilon)$ is differentiable with respect to $\varepsilon$ at $\varepsilon=0$ and $X_{\varepsilon}(\tau, 0)$ is the solution of the system

$$
Y^{\prime}=\Omega_{0}^{-1} J^{-1} H_{0} Y+\Omega_{0}^{-1} J^{-1}\left[H(\tau, 0)-\chi \Omega_{0}^{-1} H_{0}\right] X(\tau, 0)
$$

which vanishes at $\tau=0$. Therefore, by the variation of constants formula,

$$
X_{\varepsilon}(\tau, 0)=\Omega_{0}^{-1} \int_{0}^{\tau} e^{(\tau-u) Q_{0}^{-1} J^{-1} H_{0}} J^{-1}\left[H(u, 0)-\chi \Omega_{0}^{-1} H_{0}\right] e^{u Q_{0}^{-1} J^{-1} H_{0}} d u .
$$

The stability of the system (3) is determined essentially by the eigenvalues of the monodromy matrix $X(2 \pi, \varepsilon)$. If $C$ is the matrix with columns $f_{1}, \cdots, f_{n}$ then

$$
C^{-1} X(2 \pi, 0) C=e^{2 \pi 0^{-1} D}
$$

where $D=\left[i \omega_{1}, \cdots, i \omega_{n}\right]$. Hence

where

$$
C^{-1} X(2 \pi, \varepsilon) C=e^{2 \pi D^{-1} D}+\varepsilon B+o(\varepsilon),
$$

$$
\begin{aligned}
B & =C^{-1} X_{\varepsilon}(2 \pi, 0) C \\
& =\Omega_{0}^{-1} e^{2 \pi \Omega^{-1} D} \int_{0}^{2 \pi} e^{-u \Omega^{-1} D}\left[C^{-1} J^{-1} H(u, 0) C-\chi \Omega_{0}^{-1} D\right] e^{u \Omega^{-1} D} d u .
\end{aligned}
$$

But $C$ is $J$-unitary, so that $C^{-1} J^{-1}=J^{-1} C^{*}$. Therefore

$$
B=\int_{0}^{2 \pi} \Omega_{0}^{-1} e^{2 \pi \Omega_{0}^{-1} D}\left\{e^{-\tau Q^{-1} D} J^{-1} C^{*} H(\tau, 0) C e^{\tau \Omega_{0}^{-1} D}-\chi \Omega_{0}^{-1} D\right\} d \tau .
$$

Now the $(j, k)^{\text {th }}$ element of the matrix $C^{*} H(\tau, 0) C$ is the scalar product $\left(H(\tau, 0) f_{k}, f_{j}\right)$. Hence the $(j, k)^{\text {th }}$ element of the matrix in the integrand in $(4)$ is

$$
\beta_{j k}(\tau)=-i \Omega_{0}^{-1} e^{2 \pi \Omega_{0}^{-1} i \omega_{j}}\left\{\alpha_{j} e^{\tau \Omega^{-1} i\left(\omega_{k}-\omega_{j}\right)}\left(H(\tau, 0) f_{k}, f_{j}\right)+\chi \Omega_{0}^{-1} \omega_{j} \delta_{j k}\right\},
$$

where $\alpha_{j}=1$ for $j \leqq p$ and $=-1$ for $j>p$.

Let the Fourier expansion of $H(\tau, 0)$ be

$$
H(\tau, 0) \sim \sum_{s=-\infty}^{\infty} H^{(s)} e^{i s \tau}
$$

Since $H(\tau, 0)$ is Hermitian we have $H^{(-s)}=H^{(s) *}$. If $\omega_{k}=\omega_{j}+h \Omega_{0}$ for some integer $h$ the value of the integral of $\beta_{j k}(\tau)$ over the interval $(0,2 \pi)$ is

$$
b_{j k}=-2 \pi i \Omega_{0}^{-1} e^{2 \pi \Omega^{-1} i \omega_{j}}\left\{\alpha_{j}\left(H^{(-h)} f_{k}, f_{j}\right)+\chi \Omega_{0}^{-1} \omega_{j} \delta_{j k}\right\} .
$$


So far our argument has been completely general. We now restrict attention to the case mentioned at the outset and suppose that

$$
\omega_{j} \equiv \omega_{k}\left(\bmod \Omega_{0}\right)
$$

holds for $j=1, k=n$ and for no other values of $j, k$ with $j \leqq p<k$. Moreover we put

$$
m=\left(\omega_{n}-\omega_{1}\right) / \Omega_{0} .
$$

The stability of the system (3) is determined by the two eigenvalues of $X(2 \pi, \varepsilon)$ in the neighbourhood of $\lambda=e^{2 \pi i \omega_{1} / \rho_{0}}$. By the lemma and its proof these eigenvalues have the form $\lambda+\varepsilon \mu_{j}+o(\varepsilon)$, where $\mu_{1}$ and $\mu_{2}$ are the eigenvalues of the $2 \times 2$ matrix

$$
\left(\begin{array}{ll}
b_{11} & b_{1 n} \\
b_{n 1} & b_{n n}
\end{array}\right)
$$

It follows that if $\rho_{1}, \rho_{2}$ are the eigenvalues of the matrix

$$
R=\left(\begin{array}{cc}
\left(H^{(0)} f_{1}, f_{1}\right)+\chi \Omega_{0}^{-1} \omega_{1} & \left(H^{(-m)} f_{n}, f_{1}\right) \\
-\left(H^{(m)} f_{1}, f_{n}\right) & -\left(H^{(0)} f_{n}, f_{n}\right)+\chi \Omega_{0}^{-1} \omega_{n}
\end{array}\right)
$$

then the eigenvalues of $X(2 \pi, \varepsilon)$ near $\lambda$ have the form

$$
\lambda e^{-\varepsilon 2 \pi t \rho_{j} / D_{0}+\theta(\varepsilon)} \quad(j=1,2) .
$$

The quadratic equation with roots $\rho_{1}, \rho_{2}$ has real coefficients and discriminant

where

$$
\begin{aligned}
\Delta & =\left\{\left(H^{(0)} f_{1}, f_{1}\right)+\left(H^{(0)} f_{n}, f_{n}\right)-m \chi\right\}^{2}-4\left|\left(H^{(m)} f_{1}, f_{n}\right)\right|^{2} \\
& =m^{2}\left(\chi-\chi_{1}\right)\left(\chi-\chi_{2}\right) \text { if } m \neq 0,
\end{aligned}
$$

$$
\left.\chi_{1,2}=m^{-1}\left\{\left(H^{(0)} f_{1}, f_{1}\right)+\left(H^{(0)} f_{n}, f_{n}\right) \pm 2 \mid H^{(m)} f_{1}, f_{n}\right) \mid\right\} .
$$

If $\chi$ lies inside the interval $\left(\chi_{1}, \chi_{2}\right)$ then $\Delta<0$. Hence $\rho_{1}, \rho_{2}$ are not real and (3) is unstable for small $\varepsilon \neq 0$. If $\chi$ lies outside the interval $\left(\chi_{1}, \chi_{2}\right)$ then $\Delta>0$. Hence $\rho_{1}, \rho_{2}$ are real and distinct. Since $\rho_{1} \neq \rho_{2}$ the corresponding eigenvalues of $X(2 \pi, \varepsilon)$ cannot be mutually inverse with respect to the unit circle for small $\varepsilon \neq 0$ and therefore each must lie on the unit circle. Again, since $\rho_{1} \neq \rho_{2}$, each is simple. Therefore the system (3) is stable. Thus if $m \neq 0$ the system (3) is unstable or stable for small $\varepsilon \neq 0$ according as $\chi$ lies inside or outside the interval $\left(\chi_{1}, \chi_{2}\right)$ determined by (5), (6), (7).

If $m=0$ the same argument shows that, for any $\chi$, the system (3) is stable or unstable for small $\varepsilon \neq 0$ according as

$$
\left|\left(H^{(0)} f_{1}, f_{1}\right)+\left(H^{(0)} f_{n}, f_{n}\right)\right| \gtrless 2\left|\left(H^{(0)} f_{1}, f_{n}\right)\right| \text {. }
$$

In particular, we certainly have stability if $H^{(0)}>0$. 


\section{References}

[1] W. A. Coppel and A. Howe, 'On the stability of linear canonical systems with periodic coefficients', J. Austral. Math. Soc. 5 (1965), $169-195$.

[2] J. K. Hale, 'On the behavior of the solutions of linear periodic differential systems near resonance points', Contributions to the theory of nonlinear oscillations, Vol. 5, pp. 55-89. Annals of Mathematics Studies, Princeton, 1960.

[3] V. A. Jakubovic, 'On the dynamic stability of elastic systems' (Russian), Dokl. Akad. Nauk SSSR 121 (1958), 602-605.

[4] V. A. Jakubovix, 'The small parameter method for canonical systems with periodic coefficients', J. Appl. Math. Mech. 23 (1959), 17-43.

[5] M. G. Krein and V. A. Jakubovic, 'Hamiltonian systems of linear differential equations with periodic coefficients' (Russian), Proc. Internat. Sympos. Nonlinear Vibrations, Izdat. Akad. Nauk Ukrain. SSR, Kiev, 1963, Vol. 1, 277-305.

[6] N. Levinson, 'The stability of linear, real, periodic self-adjoint systems of differential equations', J. Math. Anal. Appl. 6 (1963), 473-482.

[7] I. G. Malkin, Some problems of the theory of nonlinear oscillations (Russian) (Gosud. Izdat. Tehn. Teor. Lit., Moscow, 1956).

[8] B. G. Pittel' and G. I. Juzefovič, 'Construction of domains of dynamical instability for canonical systems with periodic coefficients' (Russian), Vestnik Leningrad Univ. 17 (1) (1962), 89-101.

Department of Mathematics

Institute of Advanced Studies

The Australian National University

Canberra, A.C.T. 